\title{
THYROID NODULARITY AFTER CHILDHOOD IRRADIATION FOR LYMPHOID HYPERPLASIA: A COMPARISON OF QUESTIONNAIRE AND CLINICAL FINDINGS
}

\author{
Linda M. Pottern,,${ }^{1} *$ Michael M. Kaplan, ${ }^{2}$ P. Reed Larsen, ${ }^{3}$ J. Enrique Silva, ${ }^{4}$ \\ Ronald J. Koenig, ${ }^{5} \mathrm{~J}_{\mathrm{Ay}} \mathrm{H}$. Lubin, ${ }^{1}$ Marilyn Stovall ${ }^{6}$ and John D. Boice JR'

\begin{abstract}
${ }^{1}$ National Cancer Institute, Bethesda, MD 20892, ${ }^{2} 29877$ Telegraph Rd, Southfield, MI 48034, ${ }^{3}$ Howard Hughes Medical Institute Laboratory, Brigham and Women's Hospital, 75 Francis Street, Boston, MA 02115, ${ }^{4}$ Beth Israel Hospital, 330 Brookline Ave, Boston, MA 02215, '5ivision of Endocrinology, University of Michigan, Ann Arbor, MI 48109 and ${ }^{6}$ University of Texas, MD Anderson Cancer Center, Department of Radiation Physics, Houston, TX 77030, U.S.A.
\end{abstract}

(Received in revised form 18 September 1989)

\begin{abstract}
Ionizing radiation is a well-established cause of thyroid cancer and nodularity, however, important questions relating to the magnitude of the risk following low-dose medical exposures remain unresolved. To address these issues, we conducted a follow-up study of 1590 individuals treated between 1938 and 1969 with X-rays for childhood lymphoid hyperplasia (av. thyroid dose $=24 \mathrm{cGy}$ ) and 1499 individuals treated with surgery only. Thyroid nodularity was determined from self-administered questionnaires completed by 1195 irradiated and 1063 surgically-treated subjects and from clinical examinations of 602 irradiated and 457 non-irradiated subjects. A much higher relative risk $(\mathrm{RR})$ for radiation-induced thyroid nodules was estimated from the questionnaire than from the clinical examination data, 15.8 and 2.7, respectively. (The corresponding estimates of excess RR per cGy were 64 and 7\%). Analysis of the examination data revealed a strong dose-response relationship, similar excess RR/cGy for males and females, and an inverse relationship with age at exposure. Although the thyroid gland is one of the most sensitive organs to the neoplastic effects of radiation, the radiation-induced risk of thyroid nodularity reported from questionnaire studies may over-estimate the true risk.
\end{abstract}

Thyroid gland Thyroid nodularity Lymphoid hyperplasia Dose-response

\section{INTRODUCTION}

The thyroid gland is highly sensitive to the tumorigenic effects of ionizing radiation. Individuals irradiated in childhood for thymic enlargement [1, 2], tinea capitis [3-5], and a variety of other benign head and neck conditions [6-10]

\footnotetext{
*All correspondence should be addressed to: Dr L. M Pottern, National Cancer Institute, Epidemiology and Biostatistics Program, Executive Plaza North, Room 418, Bethesda, MD 20892, U.S.A.
}

Ionizing radiation Risk Cohort

are at increased risk for thyroid cancer or nodules after a sufficient length of time has elapsed. Excess thyroid neoplasms have also been observed among atomic bomb survivors [11] and among Marshallese Islanders accidentally exposed to radioactive fallout $[12,13]$, although patients given diagnostic doses of radioactive iodine [14] have not shown an excess. Despite these numerous studies, important questions remain as to the magnitude of the risk of thyroid tumors following low-dose medical 
$\mathrm{X}$-irradiation. Problems with existing estimates of risk include imprecise assessment of the dose to the thyroid gland, under-diagnosis of thyroid tumors, over-aggressive clinical detection of "occult" or indolent cancers among irradiated individuals, and the absence of appropriate comparison groups.

A historical prospective study was designed to overcome the limitations of previously conducted studies and to evaluate more accurately the risk of thyroid tumors and other clinical outcomes due to childhood X-irradiation for lymphoid hyperplasia, primarily enlarged tonsils or adenoids. A cohort of individuals who received radiotherapy for lymphoid hyperplasia and a non-irradiated comparison group who received only surgical treatment at the Children's Hospital Medical Center (CHMC) in Boston, Massachusetts comprised the study population. The availability of detailed radiotherapy records on virtually all the irradiated subjects enabled the reconstruction of the radiation dose to the thyroid gland for each individual.

Unlike previously conducted studies of irradiated populations, the present study included both a questionnaire and a clinical examination component for the exposed and non-exposed subjects. This design allowed for evaluation of (1) the risk of radiation-induced thyroid cancer, thyroid nodules and other head and neck conditions based on questionnaire data; (2) the risk of radiation-induced palpable thyroid nodules and thyroid dysfunction based on clinical examinations; and (3) the direct comparison of risks obtained from these two different methods of tumor ascertainment.

This paper contrasts the findings of radiationinduced thyroid nodularity determined from questionnaire and clinical examination data and explores the modifying effects of host factors such as age at exposure, gender and Jewish ethnicity on the radiogenic risk of palpable thyroid nodules.

\section{METHODS}

\section{Identification of cohort}

The study cohort consisted of individuals treated prior to age 18 with X-rays or surgery for lymphoid hyperplasia at CHMC during the years 1938-1969. The majority of the radiation treatments, $97 \%$, were for enlarged tonsils or adenoids. Figure 1 presents the number of subjects included in each step and phase of the study.

All individuals who received X-ray treatment for lymphoid hyperplasia (exposed group) were identified from radiotherapy index cards filed by diagnosis and located in the Radiation Therapy Department at CHMC. Prior to the start of this study, it was known that this index file included patients who were irradiated for lymphoid hyperplasia because researchers at CHMC and Harvard Medical School [15] conducted a questionnaire survey of the parents of these patients

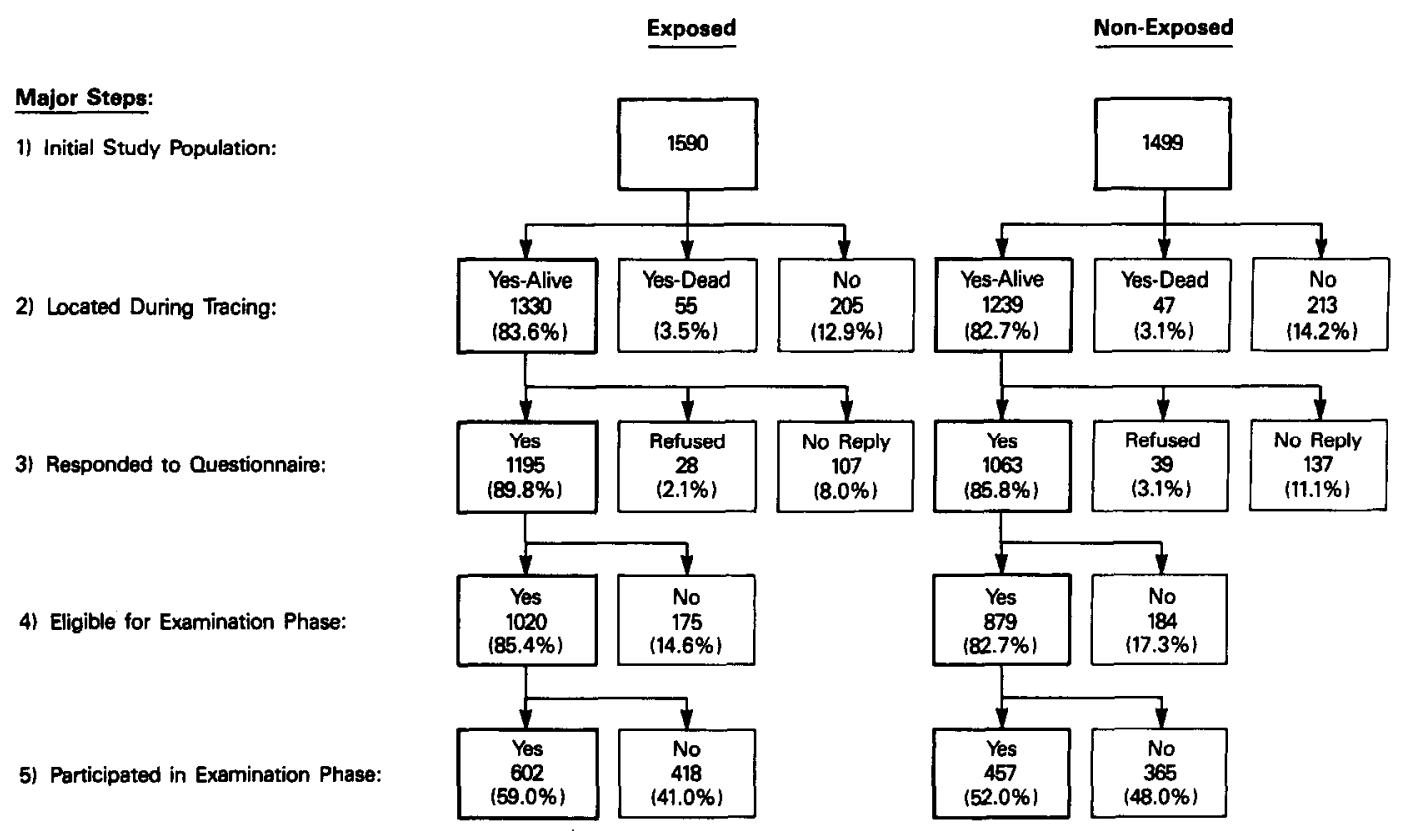

Fig. 1. Number of subjects included in each step of the study by exposure status. 
in 1958 to assess the effectiveness of such radiation treatments.

Exposed subjects were excluded from the study cohort if their medical records revealed that they (1) died or developed a thyroid disease or cancer prior to or within 1 year after their first radiation treatment at CHMC or (2) received other radiotherapy to the head or neck for a condition other than lymphoid hyperplasia. The exposed cohort consisted of 1590 ( 970 male and 620 female) X-irradiated subjects (see Fig. 1; step 1).

Surgical index cards located in the Medical Records Department at CHMC were used to identify all individuals who had a tonsillcctomy and/or adenoidectomy for treatment of lymphoid hyperplasia. A stratified random sample of these non-irradiated surgically-treated individuals (non-exposed group) was then selected based on the gender, age and year of treatment distribution of the exposed population. Nonexposed subjects were excluded from the study cohort if their medical records indicated that they (1) died or developed thyroid disease or any cancer prior to or within 1 year of their head and neck surgery at CHMC or (2) received any radiation therapy to the head or neck region. A total of 1499 (905 male and 594 female) surgically-treated individuals comprised the non-exposed cohort (see Fig. 1; step 1).

\section{Medical record abstraction}

Medical records were abstracted for demographic characteristics, past medical history and information that could provide leads for locating the subjects. An indepth review of a sample of records was undertaken to determine whether the referring physician or radiologist indicated why the lymphoid hyperplasia patient was chosen to have radiotherapy rather than surgery. This review did not indicate any specific selection criteria for a particular treatment. Patients who underwent radiotherapy did not appear to have any contraindications for surgery, in fact, the majority of them had tonsillectomies at a later date. The records of the surgically-treated only patients did not state any reason why surgery was chosen over radiotherapy. A pediatric radiotherapist who treated many of the patients in the early years informed us that radiation therapy for lymphoid hyperplasia was a common and relatively simple mode of treatment back in the early 1940 s and 1950 s and that it was at the discretion of the physician to select the appropriate type of treatment. To his knowledge, there were no established criteria for choosing one therapy over another.

\section{Radiation dosimetry}

CHMC radiation treatment records of exposed subjects were retrieved and abstracted for the following information: reason for irradiation, age at treatment, dates of treatments, regions irradiated, air doses and radiation field sizes. The nasopharyngeal region was the typical site for the X-ray treatments. The majority of the subjects received a left-sided and right-sided treatment to the neck or face on the same day. This procedure would typically be repeated a week later. The average cumulative air dose of radiation received was $800 \mathrm{R}$. The most common irradiation field sizes were $6 \times 8 \mathrm{~cm}$, $5 \times 7 \mathrm{~cm}$ and $6 \times 7 \mathrm{~cm}$. Dose to the thyroid for each individual in the study was determined using treatment details abstracted from the radiotherapy record and the measurements described below. Measurements of radiation absorbed dose were made in an anthropomorphic phantom, the size of a 6-year-old child, composed of three tissues; real skeleton and simulated lung and muscle. Treatments were simulated using $6 \times 7 \mathrm{~cm}$ lateral fields located on the nasopharynx. The phantom was irradiated using a Philips RT250 orthovoltage machine. Measurements were made at two different $\mathrm{X}$-ray energies, $3.0 \mathrm{~mm} \mathrm{Cu}$ half-value layer (HVL) and $1.2 \mathrm{~mm} \mathrm{Cu} \mathrm{HVL}$. Thermoluminescent dosimeters (TLD) were placed throughout the thyroid in order to estimate average dose to the organ. The TLD were calibrated using an ionization chamber traceable to the National Bureau of Standards. A correction factor was applied to the thyroid dose determined from the 6-year-old child to estimate dose for children of other ages. This factor was based on the distance from the field edge to the midpoint of the thyroid for children of various ages and measured water phantom data.

In general, the thyroid doses for this study are subject to an uncertainty of approximately $\pm 50 \%$. The Philips RT250 is typical of the machines used since the early 1950 s but may differ from machines used in previous years. The motion of children during treatment is also an important consideration in dose uncertainties. Any movement producing a $1 \mathrm{~cm}$ change in distance from the field edge to the thyroid gland 
results in a minimum of $\pm 30 \%$ change in the dose to the thyroid. In addition, all children were assumed to be of normal size for their ages. The uncertainty in the TLD system is $\pm 5 \%$.

\section{Location methods}

Various sources (e.g. Registry of Motor Vehicles, Town Registrar's List of Residents, Post Offices) were used during tracing until a valid address could be obtained or the subject was determined to be deceased.

Eighty-seven percent of the subjects (1385 exposed and 1286 non-exposed) were located (see Fig. 1; step 2). These subjects were similar to those not located with respect to exposure status, gender, treatment age, year of treatment, and radiation dose to the thyroid for exposed individuals.

\section{Questionnaire component}

The 2569 subjects located alive were mailed an explanatory letter and a questionnaire to complete. $90 \%$ of the exposed subjects and $86 \%$ of the non-exposed subjects completed the questionnaire (see Fig. 1; step 3). At the time of questionnaire completion, $60 \%$ of both the exposed and non-exposed questionnaire respondents lived in Massachusetts and 14\% of the exposed and $12 \%$ of the non-exposed subjects resided in other New England states (i.e. Rhode Island, Connecticut, Maine, Vermont, New Hampshire).

The questionnaire included information on: sociodemographic characteristics such as gender, birthdate, religion, education and usual occupation; medical history of thyroid disorders and cancer, thyroid medication use, head and neck operations and irradiation, and major medical conditions; and family history of thyroid conditions and cancer.

The section on thyroid disorders listed thyroid conditions such as hypothyroidism, hyperthyroidism, thyroid nodules and thyroid cancer. The subject was asked to check yes or no for each condition, record the age when the disorder was first diagnosed and the name and address of the doctor (or hospital) who treated the condition. Validation of all cancers, benign tumors, goiters, thyroid nodules, non-specific tumors or cysts reported on the questionnaire was attempted by requesting pathology reports and/or medical records from the appropriate treatment facility or physician.

\section{Clinical examination component}

All questionnaire respondents who lived in the New England area and those respondents who checked yes on the questionnaire that they planned to visit the New England area were considered eligible to participate in the examination component of the study (see Fig. 1; step 4). Cost and logistical considerations for the subjects dictated the need to place this geographic restriction on eligibility for examination.

Fach of the eligible subjects was sent a letter offering a free clinical examination, primarily of the head and neck region. $59 \%$ of the exposed and $52 \%$ of the non-exposed eligible subjects accepted our invitation (see Fig. 1; step 5). Among the questionnaire respondents who were from Massachusetts, $67 \%$ of the exposed and $62 \%$ of the non-exposed subjects were examined. $51 \%$ of the exposed and $31 \%$ of the non-exposed respondents who lived in the other New England states also agreed to the clinical examination. Of the questionnaire respondents who lived outside of New England but were considered eligible for examination because they planned to visit the area, $29 \%$ of the exposed and $20 \%$ of the non-exposed subjects actually came for an examination.

The examination sessions were held between November 1981 and October 1983. Subjects were asked to read and sign a consent form and complete a pre-examination questionnaire. The questionnaire requested current medical information and medication use that could help in interpreting the clinical examination and the blood test results. All subjects were paid U.S. $\$ 10.00$ for transportation and other expenses.

Comparisons of various sociodemographic characteristics reported on the questionnaire revealed that examined subjects were similar to subject who were not examined with respect to gender, educational status, and usual occupation. Examined and not-examined subjects also reported a similar history of thyroid disease. Among the exposed subjects, approximately $4 \%$ of both the examined subjects and not-examined subjects reported a previous diagnosis of a thyroid nodule or thyroid cancer. Among the non-exposed subjects, 1 examined subject and 2 not-examined subjects reported a history of a thyroid nodule. No thyroid cancer was reported by any non-exposed subject.

Prior to the head and neck examination, height, weight, pulse and blood pressure 
measurements were taken by a technician. In addition, $20 \mathrm{ml}$ of blood were drawn from consenting subjects for analysis of thyroid hormone concentrations and related measurements.

Each subject was examined independently by two of four collaborating thyroidologists who were unaware of the subject's exposure status and medical history. Procedures used were similar to a recent study conducted in Boston of patients exposed to multiple fluoroscopic $\mathrm{X}$-rays during tuberculosis treatments [16]. The examination included inspection of the oropharynx and palpation of the parotid glands, submandibular glands, cervical lymph node chains, and the thyroid gland. The two examiners then compared their results and if any discrepancies existed they reexamined the subject and tried to reach a consensus. The consensus findings were recorded on a standardized summary form and explained to the subject.

During the design of the clinical examination component of the study, the hospital medical director, the collaborating thyroidologists and the epidemiologists from the National Cancer Institute met and discussed whether thyroid scintiscans should be given as a routine procedure (i.e. screening) to all subjects undergoing a thyroid examination. Since the clinical significance of "occult" thyroid tumors detected only by scintiscans is not clcarly understood, it was decided that thyroid scans should be used only as an aid for interpreting the palpation findings.

A subject was recommended to have a pertechnetate thyroid scintiscan (free of charge) only if the thyroidologist detected a definite or uncertain palpable thyroid nodule during the clinical examination. The scan was not performed if the subject reported having one within the past 6 months, was currently taking thyroid hormone medication or was pregnant. Of the 86 study subjects for whom a scintiscan was recommended, 64 had the scan. The scintiscans were read by the examiner who compared them with the recorded palpation findings with respect to the thyroid size and shape, and to the presence, location and focal or diffuse nature of scintigraphic abnormalities. An uncertain palpable nodule was reclassified as a definite nodule if there was a scan abnormality in the corresponding anatomical location. After evaluation of the scan findings, the examiners changed the diagnosis of an uncertain palpable nodule to a definite nodule for 11 exposed and 2 non-exposed subjects.
The results of the head and neck examination were sent to the study subject and/or the subject's physician. Specific treatment recommendations were not suggested but a list of available consulting specialists was offered. Since the diagnosis of thyroid cancer is made primarily on the basis of clinical suspicion and pathological confirmation, it was necessary to obtain treatment follow-up information on all subjects who had a palpable thyroid nodule detected during the examination. A letter along with a treatment follow-up form was sent to the subject's physician to obtain information on the type of treatment the subject may have received (e.g. surgery or medication) and future follow-up plans. A pathology report was requested if the subject was surgically treated.

\section{Data analysis}

Outcome variable. Non-specific thyroid nodular disease was the outcome variable of interest in the present analysis. Thyroid cancer as a distinct entity was not evaluated since comparative analyses between questionnaire and examination findings of thyroid cancer were not possible. Clinical examinations can provide information on the presence of palpable nodules but cannot differentiate between benign and malignant thyroid tumors. A biopsy is necessary to confirm a diagnosis of thyroid cancer.

From the questionnaire data, thyroid nodularity was defined as a reported history of thyroid nodules or thyroid cancer. From the examination results, thyroid nodularity was defined as either the presence of a definite palpable thyroid nodule or a thyroidectomy scar (evidence of previous surgical removal of a thyroid tumor) at examination. Multinodular goiters were not classified as thyroid nodules.

Relative risk estimation. To conduct a comparative analysis of questionnaire and clinical examination data, it was necessary to select a uniform measure which would describe the occurrence rate of thyroid nodularity. Prevalence rather than incidence was used in the analysis since the clinical examinations would detect only prevalent cases of thyroid nodules. To measure the strength of association between $\mathrm{X}$-ray exposure for lymphoid hyperplasia and thyroid nodular disease, prevalence odds ratios, as an estimate of the relative risk (RR), and 95\% confidence intervals (CI) were calculated $[17,18]$. The RR was considered statistically significant if the confidence interval did not 
include the value one. Adjustment for potential confounder variables (e.g. gender, age at treatment) was performed using stratification techniques. To determine if strata-specific RRs differed significantly from each other, a chisquare test for homogeneity across strata was used [17]. RRs according to dose categories were calculated using the non-exposed subjects (i.e. zero exposure) as the referent group.

Dose-response models. To assess doseresponse, variants of the usual logistic regression model was modified and fit to the data. The odds of thyroid nodular disease for dose $d$ is represented by the following model:

$$
P(\mathrm{D}=1 \mid d) / P(\mathrm{D}=0 \mid d)=\exp (\alpha) R(d)
$$

where $\mathrm{D}=1$ denotes diseased and $\mathrm{D}=0$ denotes disease-free individuals, $R(d)$ is the relative odds in dose $d$, and $\exp (\alpha)$ represents the effect of variables other than dose on the odds of disease. At baseline exposure, i.e. zero dose, $R(0)=1$ so that the odds of thyroid nodules for the non-exposed subjects are $\exp (\alpha) R(0)=$ $\exp (\alpha)$. If $R$ has an exponential form $[R(d)=\exp (\beta d)]$, then model (1) is the usual logistic regression model. Our analysis indicated that the relative odds were best characterized by a linear dose-response function, that is,

$$
R(d)=1+\beta d
$$

where $d$ is the continuous dose in cGy and where $\beta$ is the linear slope parameter, interpretable as the excess RR per unit increase in dose. Other dose-response models, such as the linear exponential $[R(d)=(1+\beta d) \exp (-\tau d)]$ and the linear quadratic $\left.\left[R(d)=1+\beta d+\tau d^{2}\right)\right]$, did not fit the data significantly better than model (2). To assess whether $R(d)$ varied by any of our stratifying variables (e.g. age at treatment, year of treatment or gender), model (2) was modified to include these variables as follows;

$$
R(d, x)=1+\beta_{j} d
$$

where $x$, the stratifying variable, was divided into $J$ categories and where $\beta_{j}$ is the excess $\mathrm{RR} / \mathrm{cGy}$ within the $j$ th category. To account for differences in the odds of thyroid nodular disease due to factors distinct from radiation dose, i.e. gender $(\mathrm{g})$ (with $\mathrm{g}=1$ denoting females and $\mathrm{g}=0$ denoting males), year of treatment $(\mathrm{y})$, and age at treatment (a), we incorporated separate parameters into model (1) as follows:

$$
\exp (\alpha+\theta \mathrm{g}+\phi \mathrm{y}+\tau \mathrm{a}) R(d)
$$

where $\theta, \phi, \tau$ are unknown parameters and where the dose effect is again given by model (2) or model (3). Since Jewish ethnicity has been suggested as a risk factor for thyroid tumors, we also added religion (Jewish and other) into model (4) as a main effect and a modifier of dose, but neither effect was significant. To test whether strata-specific excess odds ratios differed significantly from each other, a chisquare test of homogeneity with $J-1 d f$ was calculated by taking twice the difference between the maximum log-likelihoods of models (2) and (3).

\section{RESULTS}

\section{General characteristics of study participants}

The characteristics of the questionnaire respondents and examined subjects by exposure status are shown in Table 1. For both the questionnaire respondents and examined subjects, there were no statistically significant differences between the exposed and nonexposed groups for any of the sociodemographic variables thought to be related to either exposure or disease outcome (i.e. gender, race, religion, education, occupation). Both exposed and non-exposed subjects were highly educated with over $77 \%$ having attended college and more than $45 \%$ reporting a usual occupation in the managerial or professional category. Exposed subjects were slightly older than nonexposed subjects at time of treatment (i.e. X-ray or surgery). The average years of follow-up were 29 for all subjects with a maximum length of 44 and 47 years for exposed and non-exposed subjects, respectively. For the exposed subjects, the mean thyroid dose was $24 \mathrm{cGy}$.

\section{Comparative risk estimates for thyroid nodular disease}

Table 2 presents the RRs and percent distribution of thyroid nodular disease calculated from questionnaire data and examination data. Among the exposed subjects, $4.3 \%$ of the questionnaire respondents reported a history of thyroid nodularity, whereas $10.3 \%$ of the examined subjects had a palpable thyroid nodule or a thyroidectomy scar. Among the nonexposed subjects, $0.3 \%$ of the questionnaire respondents reported thyroid nodules, while in contrast, $4.2 \%$ of the exam participants had a palpable thyroid nodule or a thyroidectomy scar. The radiation-induced RR of thyroid nodularity calculated from questionnaire data 
Table 1. Characteristics of questionnaire respondents and clinical examination participants by exposure status

\begin{tabular}{|c|c|c|c|c|}
\hline \multirow[b]{2}{*}{ Characteristic } & \multicolumn{2}{|c|}{ Questionnaire respondents } & \multicolumn{2}{|c|}{ Examined participants } \\
\hline & $\begin{array}{c}\text { Exposed } \\
(n=1195)\end{array}$ & $\begin{array}{l}\text { Non-exposed } \\
\quad(n=1063)\end{array}$ & $\begin{array}{l}\text { Exposed } \\
(n=602)\end{array}$ & $\begin{array}{c}\text { Non-exposed } \\
(n=457)\end{array}$ \\
\hline & \multicolumn{4}{|c|}{ Number of subjects (\%) } \\
\hline Sex: female & $465(38.9)$ & $432(40.6)$ & $223(37.0)$ & $187(40.9)$ \\
\hline Race: white & $1166(97.6)$ & $1037(97.6)$ & $587(97.5)$ & $449(98.3)$ \\
\hline Religion: Jewish & $144(12.1)$ & $155(14.6)$ & $82(13.6)$ & $69(15.1)$ \\
\hline Education: college & $940(78.7)$ & $863(81.2)$ & $465(77.2)$ & $328(80.5)$ \\
\hline Marital status: married & $778(65.1)$ & $717(67.5)$ & $390(64.8)$ & $322(70.5)$ \\
\hline \multirow[t]{2}{*}{ Usual occupation: managerial/professional } & $546(45.7)$ & $515(48.5)$ & $269(44.7)$ & $215(47.1)$ \\
\hline & \multicolumn{4}{|c|}{$\begin{array}{l}\text { Mean } \pm S D \\
\text { (range) }\end{array}$} \\
\hline Year of treatment & $\begin{array}{r}1952.7 \pm 5.2 \\
(1938-69)\end{array}$ & $\begin{array}{r}1952.8 \pm 4.3 \\
(1935-66)\end{array}$ & $\begin{array}{r}1952.8 \pm 5.1 \\
(1938-68)\end{array}$ & $\begin{array}{r}1952.9 \pm 4.3 \\
(1937-64)\end{array}$ \\
\hline Year of birth & $\begin{array}{r}1945.8 \pm 5.7 \\
(1926-61)\end{array}$ & $\begin{array}{r}1946.5 \pm 4.5 \\
(1929-65)\end{array}$ & $\begin{array}{r}1945.9 \pm 5.5 \\
(192661)\end{array}$ & $\begin{array}{r}1946.4 \pm 4.3 \\
(1931-60)\end{array}$ \\
\hline Age at treatment $(\mathrm{yr})$ & $\begin{array}{r}6.9 \pm 2.8 \\
(0.4-17.3)\end{array}$ & $\begin{array}{r}6.2 \pm 2.7 \\
(0.4-16.0)\end{array}$ & $\begin{array}{r}6.9 \pm 2.8 \\
(0.4-16.0)\end{array}$ & $\begin{array}{r}6.4 \pm 2.8 \\
(0.4-16.0)\end{array}$ \\
\hline Age at follow-up (yr) & $\begin{array}{r}36.0 \pm 5.8 \\
(21.0-56.0)\end{array}$ & $\begin{array}{r}35.7 \pm 4.5 \\
(17.0-53.0)\end{array}$ & $\begin{array}{r}35.7 \pm 5.5 \\
(21.0-56.0)\end{array}$ & $\begin{array}{r}35.7 \pm 4.4 \\
(23.0-52.0)\end{array}$ \\
\hline Years of follow-up & $\begin{array}{r}29.1 \pm 5.3 \\
(12.0-44.0)\end{array}$ & $\begin{array}{r}29.4 \pm 4.3 \\
(16.0-47.0)\end{array}$ & $\begin{array}{r}28.8 \pm 5.2 \\
(12.0-44.0)\end{array}$ & $\begin{array}{r}29.2 \pm 4.3 \\
(18.0-46.0)\end{array}$ \\
\hline Thyroid dose (cGy) & $\begin{array}{l}24.1 \pm 6.6 \\
(3.2-55.0)\end{array}$ & 一 & $\begin{array}{l}24.2 \pm 6.6 \\
(3.2-53.0)\end{array}$ & - \\
\hline
\end{tabular}

$(\mathrm{RR}=15.8)$ was almost six times greater than the RR calculated from the examination data $(\mathrm{RR}=2.7)$.

Among the 51 exposed questionnaire respondents who reported a history of thyroid nodular disease, 11 had a history of surgical removal of thyroid cancer, four of which had a simultaneous diagnosis of thyroid cancer and thyroid nodule at time of surgery. Of the remaining 40 exposed subjects who reported having thyroid nodules, 27 had surgical removal of the nodule, 8 were given thyroid medication only, 3 received no treatment and 2 did not report the type of treatment. No non-exposed subject reported a history of thyroid cancer. Of the 3 non-exposed subjects with a history of thyroid nodules, 2 had surgical removal and 1 had no treatment.

For the 41 exposed subjects who had a palpable thyroid nodule detected during the clinical examination (excluding the 21 subjects with a thyroidectomy scar), it was determined through contacts with the subjects' physicians that 11 underwent surgery within 1 year of detection. Two of these individuals had pathological confirmation of thyroid cancer; papillary and mixed papillary-follicular carcinoma. Among the 17 non-exposed subjects found to have a thyroid nodule during palpation (excluding the 2 subjects with a thyroidectomy scar), 5 had subsequent surgical treatment. Two of these subjects had thyroid cancer; papillary and mixed papillary-follicular carcinoma.

\section{Dose-response relationship}

An increase in the RR for thyroid nodular disease was seen with increasing radiation dose to the thyroid for both questionnaire respondents and examined subjects (Table 3 ). The

Table 2. Relative risks (RR) of thyroid nodular disease associated with prior radiotherapy based on questionnaire and clinical examination data

\begin{tabular}{|c|c|c|c|c|c|c|c|c|}
\hline & \multicolumn{4}{|c|}{ Questionnaire data } & \multicolumn{4}{|c|}{ Examination data* } \\
\hline & $\begin{array}{c}\text { Exposed } \dagger,+ \\
n=1192 \\
\text { No. }(\%)\end{array}$ & $\begin{array}{c}\text { Non-exposed } \S \\
n=1063 \\
\text { No. }(\%)\end{array}$ & RR & $(95 \% \mathrm{Cl})$ & $\begin{array}{c}\text { Exposed } \\
n=602 \\
\text { No. }(\%)\end{array}$ & $\begin{array}{c}\text { Non-exposed } \\
n=457 \\
\text { No. }(\%)\end{array}$ & RR & $(95 \% \mathrm{CI})$ \\
\hline \multicolumn{9}{|c|}{ Nodular disease } \\
\hline No & $1141(95.7)$ & $1060(99.7)$ & 1.0 & - & $540(89.7)$ & $438(95.8)$ & 1.0 & - \\
\hline Yes & $51(4.3)$ & $3(0.3)$ & 15.8 & $(4.7-63.5)$ & $62(10.3)$ & $19(4.2)$ & 2.7 & $(1.5-4.7)$ \\
\hline
\end{tabular}

*Includes 21 exposed and 2 non-exposed subjects with thyroidectomy scar at time of examination.

†Includes subjects with the following thyroid disorders: thyroid cancer (7), thyroid nodules (40), and thyroid cancer plus thyroid nodules (4).

$\ddagger$ Excludes 3 subjects with missing data on thyroid disorders.

§No non-exposed subjects reported thyroid cancer. 
Table 3. Relative risks (RR) of thyroid nodular disease associated with prior radiotherapy based on clinical examination data by radiation dose to the thyroid gland

\begin{tabular}{|c|c|c|c|c|c|c|c|c|}
\hline & \multicolumn{8}{|c|}{ Thyroid nodular disease } \\
\hline & \multicolumn{4}{|c|}{ Questionaire data* } & \multicolumn{4}{|c|}{ Examination data $\dagger, \ddagger$} \\
\hline & Yes & No & RR & $(95 \% \mathrm{CI})$ & Yes & No & RR & $(95 \% \mathrm{CI})$ \\
\hline \multicolumn{9}{|l|}{ Thyroid dose (cGy) } \\
\hline 0 & 3 & 1060 & 1.0 & - & 19 & 438 & 1.0 & - \\
\hline $1-19$ & 4 & 202 & 7.0 & $(1.3-39.6)$ & 11 & 89 & 2.9 & $(1.2-6.6)$ \\
\hline $20-24$ & 13 & 341 & 13.5 & $(3.6-59.8)$ & 12 & 168 & 1.7 & $(0.7-3.7)$ \\
\hline $25-29$ & 23 & 456 & 17.8 & $(5.1-74.9)$ & 28 & 216 & 3.0 & $(1.6-5.7)$ \\
\hline $30-59$ & 10 & 133 & 26.6 & $(6.7-123.2)$ & 11 & 62 & 4.1 & $(1.7-9.6)$ \\
\hline Excess RR/cGy§ & & & 0.64 & $(0.18-2.25)$ & & & 0.07 & $(0.03-0.15)$ \\
\hline
\end{tabular}

*Includes thyroid nodules and thyroid cancer.

†ncludes 21 exposed and 2 non-exposed subjects with thyroidectomy scar at time of examination.

$\ddagger$ Excludes 5 subjects with missing dosimetry data.

$\S$ Excess RR/cGy $=(R R-1) / c G y$, and is adjusted for year of treatment, gender, and age at treatment; based on the linear dose-response function [text model (2)] and text model (4).

excess RR per cGy based on models (2) and (4) with adjustments for gender, age at treatment and year of treatment differed markedly between questionnaire data and examination data: $64 \%(95 \% \mathrm{CI}=20-230 \%)$ and $7 \%(95 \% \mathrm{CI}=$ $3-20 \%$ ), respectively. In as much as the examination results were assumed to be more reliable, additional analyses of these data examined the modifying effects of gender, age at treatment, religion and number of X-ray treatments on the risk of palpable thyroid nodules.

\section{Effect modification on risk for palpable thyroid nodules}

Table 4 presents the RR of thyroid nodularity by gender for exposed and non-exposed subjects. The risk was higher among women than men, for both the exposed group $(\mathrm{RR}=6.3 / 3.2=2.0)$ and non-exposed group $(R R=2.6)$. The estimated $R R$ due to radiation was similar among men $(R R=3.2)$ and women
$(\mathrm{RR}=6.3 / 2.6=2.4)$. Accordingly, the associated estimates of excess RR/cGy for men and women based on multivariate modeling procedures (text-model 3) were 7 and $6 \%$, respectively (Table 5).

Analysis of the examination data according to religious background (Jewish and other) was conducted to evaluate whether Jews are at an increased risk of thyroid nodules and are more susceptible to radiation-induced nodularity (Table 4). Interestingly, the risk was higher among Jews than non-Jews for the exposed group $(\mathrm{RR}=3.8 / 2.0=1.9)$ but not for the nonexposed group $(\mathrm{RR}=0.3)$. Furthermore, the estimates of excess RR/cGy for Jews and nonJews were 39 and $5 \%$, respectively (Table 5). It should be noted, however, that the exceptionally high radiation risk among Jews was related more to an exceptionally low risk among the non-exposed subjects than to an exceptionally elevated risk among the exposed subjects.

Table 4. Relative risks (RR) of thyroid nodular disease associated with prior radiotherapy based on clinical examination data by gender and religion for exposed and non-exposed subjects

\begin{tabular}{lrrrc}
\hline & \multicolumn{5}{c}{ Thyroid nodular disease } \\
\cline { 2 - 5 } & Yes & No & RR & $(95 \% \mathrm{CI})$ \\
\hline Gender & 7 & 263 & 1.0 & - \\
$\quad$ Males, non-exposed & 12 & 175 & 2.6 & $(0.9-7.4)$ \\
Females, non-exposed & 30 & 349 & 3.2 & $(1.3-8.2)$ \\
Males, exposed & 32 & 191 & 6.3 & $(2.6-16.0)$ \\
Females, exposed & & & & \\
Religion*, $\dagger$ & 17 & 341 & 1.0 & - \\
$\quad$ Non-Jewish, non-exposed & 1 & 68 & 0.3 & $(0.0-2.2)$ \\
Jewish, non-exposed & 44 & 432 & 2.0 & $(1.1-3.8)$ \\
Non-Jewish, exposed & 13 & 69 & 3.8 & $(1.6-8.7)$ \\
Jewish, exposed & &
\end{tabular}

*Excludes 30 non-exposed subjects with missing data on religion; 1 with and 29 without thyroid nodular disease.

†Excludes 44 exposed subjects with missing data on religion; 5 with and 39 without thyroid nodules. 
Table 5. Relative risks (RR) of thyroid nodular disease by dose categories for examined subjects by gender, age at treatment religion and number of radiation treatments

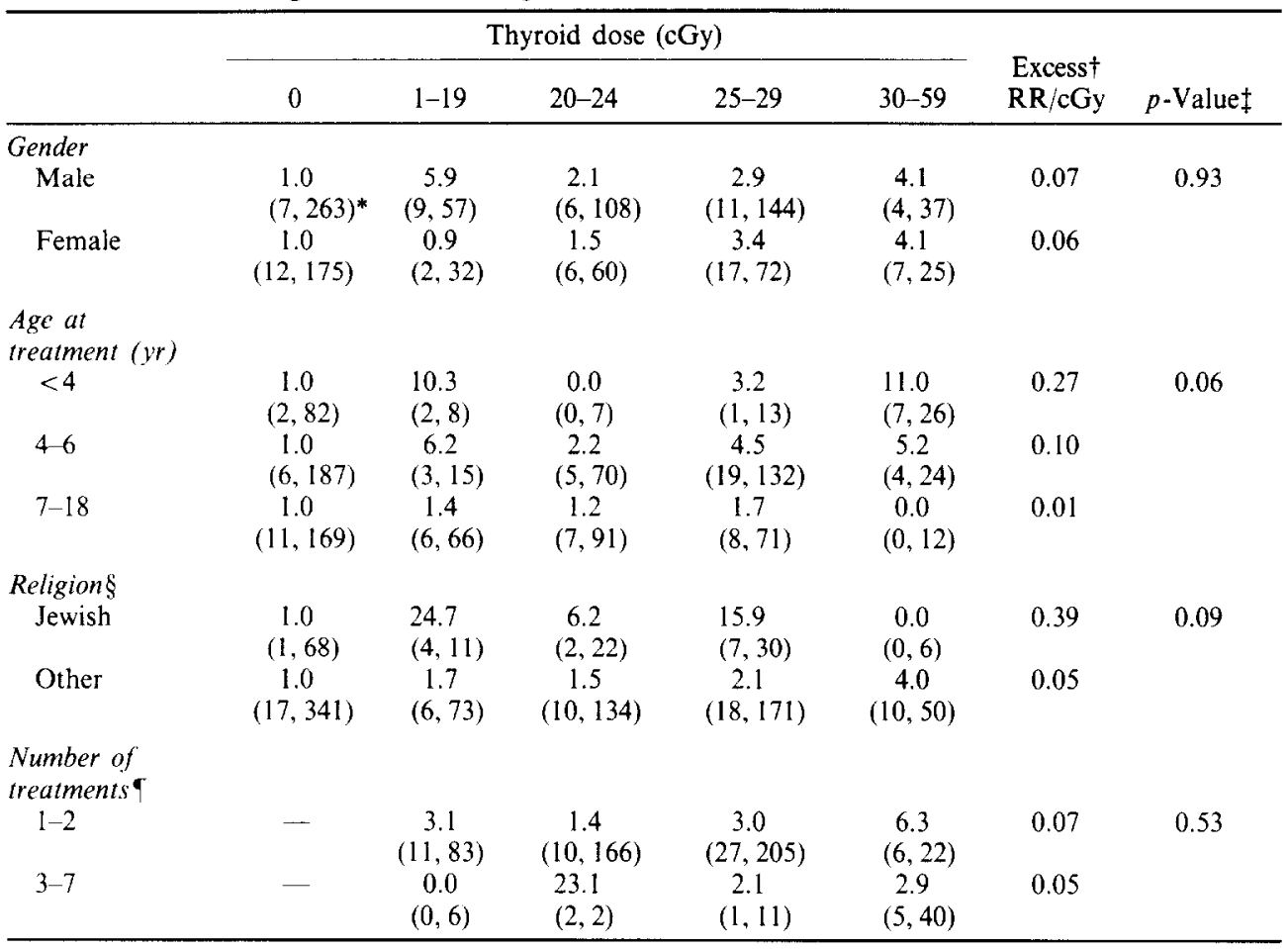

*Numbers in parentheses indicate the number of subjects with and without thyroid nodules.

+ Excess $R R / c G y=(R R-1) / c G y$, is adjusted for year of treatment, gender and age at treatment; based on text model (3) with dose continuous.

$\ddagger p$-Value for likelihood ratio test of homogeneity of linear risk parameters.

\$Model includes non-significant main effect for religion; excludes 74 subjects with unknown religion.

- $\mathrm{RR}=1$ for non-exposed subjects.

The risk of developing thyroid nodules among exposed subjects declined with age at first X-ray treatment, with subjects under age 4 years being at highest risk when compared to those aged 7-18 years ( $\mathrm{RR}=2.1, \mathrm{CI}=0.9-5.1$ ). Early age at exposure was also associated with a much greater excess RR/cGy than exposure at later ages (Table 5).

To assess whether dose fractionation decreases the risk of thyroid nodules, number of $\mathrm{X}$-ray treatment sessions (a left-sided and rightsided treatment to neck or face given on the same day constituted a session) was included in model (4). The excess RR of thyroid nodules per cGy of exposure for subjects who had less than three X-ray treatments was similar to those who had three or more treatments (Table 5).

\section{DISCUSSION}

The present study is the first to evaluate the effects of low-dose irradiation on the thyroid gland by incorporating all of the following design elements: (1) comparison of questionnaire and clinical examination results, (2) com- prehensive dosimetry on individuals and (3) the use of a comparison population treated by another modality (surgery) for the same condition as those exposed to medical irradiation. The main conclusions are that questionnaire data alone appear to overestimate the impact that prior irradiation has on thyroid nodularity, and that young age at exposure is a strong predictor of future risk. Furthermore, radiation risk on a relative scale was similar for men and women and possibly higher among Jews.

The risk of radiation-induced thyroid cancer could not be addressed since the percentage of thyroid cancers which comprised the thyroid nodular disease category was not known. 33\% of the questionnaire respondents who specifically reported a thyroid nodule did not have surgery and $80 \%$ of the examined subjects found to have a palpable thyroid nodule did not seek medical attention or did not have surgical intervention within 1 year of detection.

Little is known about the natural history of thyroid cancer and thyroid nodules. Papillary carcinoma, the most common histologic type of thyroid cancer, behaves in a clinically benign 
fashion more so than the other cell types. It is usually slow growing and typically spreads to the regional lymph nodes where it may remain indolent for many years. It is the primary histologic type of thyroid cancer induced by radiation. Solitary thyroid nodules are also typically slow growing and initially do not disturb normal thyroid function [19]. Thyroid nodules have also been shown to regress over time [20]. The relationship between thyroid nodules and thyroid cancer is unclear. Several casecontrol studies have shown that a history of thyroid nodules is a risk factor for developing thyroid cancer [21,22], and biopsies of thyroid nodules in irradiated thyroid glands often reveal a high proportion of malignancies [9]. However, the progression of a thyroid nodule to cancer has not been well-documented [23].

Our comparative analysis revealed a disparity between prevalence rates of thyroid nodular disease calculated from questionnaire and examination data. Based on questionnaire data, thyroid nodules appeared to be underascertained among both exposed and non-exposed individuals. For the exposed subjects, the ratio of the proportion of examined subjects to the proportion of questionnaire respondents with thyroid nodules was 2.4 . For the non-exposed subjects, this ratio was 14.0 . The greater underascertainment of thyroid nodules among the non-exposed individuals resulted in an almost six-fold difference in the relative risk estimates between questionnaire and examination data. Similarly, there was a nine-fold difference in the estimates of the excess RR/cGy between these two sources of data.

Underascertainment of thyroid nodules, in general, is not an unexpected finding. Thyroid nodules often follow an uneventful course and unless symptoms suggestive of thyroid disease are present, thyroid examination is usually not conducted among young adults. Two possible explanations why there was greater underascertainment among the non-exposed than the exposed subjects are: (1) exposed subjects who were aware of their childhood X-ray treatments may have approached their physician for thyroid screening or (2) physicians who knew that a subject had a history of head and neck irradiation were more likely to conduct thyroid examinations. Both of these situations could have led to greater detection of asymptomatic thyroid tumors among the exposed subjects.

The participation rates in the examination component of the study were lower than initially anticipated. Nevertheless, our data are consistent with the results from other studies and appear to reflect the "true" prevalence rates of thyroid nodular disease for both the nonexposed and exposed subjects. Among the nonexposed subjects examined in our study, $4.2 \%$ had a palpable thyroid nodule or a thyroidectomy scar at examination. This is identical to the percentage of thyroid nodules detected in the population participating in the Framingham Heart Disease Study [23] and among the nonexposed comparison group in a previous study conducted in Boston on tuberculosis patients which used examination procedures similar to our study [16].

Among the examined exposed subjects, $10.3 \%$ had a palpable thyroid nodule which is consistent with palpation results from two recall programs of exposed individuals. In the large screening program conducted at Michael Reese Hospital, Chicago [7] about $13 \%$ of the exposed subjects had a palpable thyroid nodule. This figure increased to $16.5 \%$ after the examiner reviewed the scintigram and repalpated the subject. In an areawide cooperative program of thyroid screening conducted in Milwaukee [24], $10 \%$ of the exposed subjects screened in the first year were found to have nodules.

As in any epidemiologic study which relies on a subject's willingness to be examined, there exists the potential for self-selection bias. To address this issue, we first evaluated participation rates by residential area. The majority of our study population resided in Massachusetts, of whom a similar percentage of exposed and non-exposed subjects agreed to be examined. For subjects living in the other New England states or outside the New England area, a higher percentage of eligible exposed than non-exposed subjects were examined. The prevalence rates of thyroid nodules detected during the examination by residential area and exposure status were evaluated to determine whether irradiated subjects who had convenient access to the clinic were more likely to have a palpable thyroid nodule than those who lived outside of Massachusetts. The exposed subjects who traveled from outside the New England area had essentially the same prevalence rate of palpable thyroid nodules as the exposed subjects who came from Massachusetts. It is, therefore, unlikely that self-selection bias influenced our findings. The greater cooperation rates among the exposed subjects who lived outside of Massachusetts may have been due to their awareness of 
their radiation history and potential risk for thyroid tumors.

Thyroid disease, in general, typically affects females more often than males. It has been suggested in several studies of irradiated populations that women are also more susceptible than men to radiogenic thyroid tumors $[1,3,5]$. Often there is confusion interpreting this finding because the scale upon which these inferences are based is not defined. Women have a higher risk of developing thyroid nodular disease than men, regardless of radiation history, as seen in our study. On a relative scale, however, men and women appear equally susceptible to radiationinduced thyroid nodularity. Because of the much higher underlying rate of thyroid disease among women, the finding of a similar RR due to radiation for men and women implies that women have a greater number of excess thyroid nodules (and higher absolute risk) than men.

Age at treatment has been identified as an important modifying factor in radiationinduced thyroid disease $[1,3]$. Our findings further support this conclusion. The excess risk of thyroid nodules/cGy was highest for subjects treated under 4 years of age with only a slight excess RR/cGy seen for subjects treated after age 6 . This suggests that the very young thyroid gland may be especially sensitive to radiation, possibly because of a more active cell proliferation capacity.

Jewish ethnicity has also been suggested as a susceptibility factor for radiation-induced thyroid neoplasms $[1,25]$. In our study, Jews were at a higher radiation risk for thyroid nodularity than non-Jews but this increase appeared due, a large part, to a peculiar deficit of nodules among non-exposed Jews. The reason for this finding is unclear but may be related to the small number of Jewish subjects in the study.

The exposed subjects in our study received relatively low doses of radiation to their thyroid glands and within a limited dose range (3-55 cGy; mean 24 cGy). Nevertheless, analysis of the risk of thyroid nodular disease by dose revealed a statistically significant dose-response relationship. The estimate of the percent excess $\mathrm{RR}$ of thyroid nodules/cGy was $7 \%$ for the examined subjects. Dose-response relationships for thyroid cancer and thyroid adenomas were also seen in the Israeli study of children with tinea capitis who received low doses of irradiation ( $4-50 \mathrm{cGy}$ ) to the thyroid gland, although the estimate of excess $\mathrm{RR} / \mathrm{cG}$ y was higher $(10-30 \%)[3,4]$.
Dose fractionation, measured by number of treatment sessions, did not appear to decrease the risk of thyroid nodules. Subjects who had three or more treatments had a slightly smaller excess $R R / c G y$ of thyroid nodules than the subjects who had less than three treatments. A thorough investigation of the effects of dose fractionation on the risk of thyroid nodularity, however, could not be made in this study. In order to find a fractionation effect, there should be comparable total doses for a group of subjects with and without fractionated treatments. In our study, additional fractions (treatments) indicated higher total doses thus dose fractionation could not be adequately assessed.

The latency period for the development of radiation-induced thyroid nodules cannot be addressed in this study since the clinical examination data were prevalent cases of thyroid nodules detected at a specific point in time. The time period during which the subject was disease free could not be established. Nevertheless, radiation-induced thyroid nodules were detected at least 40 years after exposure; 44 years being the maximum length of follow-up for exposed examined subjects in our study. This is consistent with findings from the Michael Reese recall program [8] and from the follow-up study of thymus-irradiated children [2].

In conclusion, the association between lowdose $\mathrm{X}$-irradiation for lymphoid hyperplasia and the development of thyroid nodules later in life was confirmed by both questionnaire and thyroid examination data on exposed and nonexposed subjects. The magnitude of the association depended on which data source was used. The questionnaire data seemed biased in that a greater proportion of non-exposed subjects had an underascertainment of thyroid nodules. We believe that clinical examinations, which include a non-exposed comparison group, provide the best risk estimates of radiation-induced thyroid nodular disease since such examinations are less susceptible to reporting and ascertainment biases associated with questionnaire surveys.

Acknowledgements--The authors wish to thank Dr John F. Crigler Jr our liaison at Children's Hospital Medical Center, Boston, for his assistance and support; Ms Susan A. Smith, at the Department of Radiation Physics at the University of Texas Systcm Cancer Centcr in Houston for assisting in the estimation of absorbed radiation dose to the thyroid gland and other organs; Ms Linda Morris Brown at NCI for her analytic advice and technical review; $\mathrm{Mr}$ Dan Ames of Westat, Inc., for his assistance in managing the study; and Ms Susan Kornetsky for coordinating the exam activities. 
The clinical examination phase was supported in part by Brigham and Women's Hospital under contract to the National Cancer Institute (NO1-CP-1 1008).

\section{REFERENCES}

1. Hempelmann LI, Hall WJ, Phillips M, Cooper RA, Ames, WR. Neoplasms in persons treated with $\mathrm{X}$-rays in infancy: Fourth survey in 20 years. $\mathbf{J}$ Natl Cancer Inst 1975; 55: 519-530.

2. Shore RE, Woodard ED, Hildreth N, Dvoretsky P, Hempelmann LH, Pasternack B. Thyroid tumors following thymus irradiation. J Natl Cancer Inst 1985; 74: 1177-1184

3. Ron E, Modan B. Benign and malignant thyroid neoplasms after childhood irradiation for tinca capitis. J Natl Cancer Inst 1980; 65: 7-11.

4. Ron E, Modan B, Preston D, Alfandary E. Stovall M, Boice JD Jr. Thyroid neoplasia following low-dose radiation in childhood. Radiat Res 1989; 120: 516-531.

5. Shore RE, Albert RE, Pasternack BS. Follow-up study of paticnts trcatcd by $x$-ray epilation for tinea capitis. Resurvey of post-treatment illness and mortality experience. Arch Environ Health 1976; 31: 21-28.

6. Saenger EL, Silverman FN, Sterling TD, Turner ME. Neoplasia following therapeutic irradiation for benign conditions in childhood. Radiology 1960; 6: 889-904.

7. Favus MJ, Schneider AB, Stachura ME, Arnold JE, Ryo UY, Pinsky SM, Colman M, Arnold MJ, Frohman LA. Thyroid cancer occurring as a late consequence of head-and-neck irradiation: Evaluation of 1056 patients. N Engl J Med 1976; 294: 1019-1025.

8. Schneider AB, Favus MJ, Stachura ME, Arnold J, Arnold MJ, Frohman LA. Incidence, prevalence and characteristics of radiation-induced thyroid tumors. Am J Med 1978; 64: 243-252.

9. Schneider AB, Shore-Freedman E, Ryo UY, Bekerman C, Favus M, Pinsky S. Radiation-induced tumors of the head and neck following childhood irradiation: Prospective studies. Medicine 1985; 64: 1-15.

10. Maxon HR, Thomas SR, Saenger EL, Buncher CR, Kereiakes JG, Ionizing irradiation and the induction of clinically significant disease in the human thyroid gland. Am J Med 1977; 63: 967-978.

11. Prentice RL, Kato $\mathrm{H}$, Yoshimoto $\mathbf{K}$, Mason $\mathbf{M}$ Radiation exposure and thyroid cancer incidence among Hiroshima and Nagasaki residents. Natl Cancer Inst Monogr 1982; 62: 207-212.

12. Conard RA. Late radiation effects in Marshall Islanders exposed to fallout 28 years ago. In: Boice JD Jr, Fraumeni JF Jr, Eds. Radiation Carcinogenesis:
Epidemiology and Biological Significance. New York: Raven Press; 1984: 57-71.

13. Hamilton TE, van Belle G, LoGerfo JP. Thyroid neoplasia in Marshall Islanders exposed to nuclear fallout. JAMA 1987; 258: 629-636.

14. Holm L-E, Wiklund KE, Lundell GE, Bergman NA, Bjelkengren G, Cederquist ES, Ericsson U-BC, Larsson L-G, Lidberg ME, Lindberg RS, Wicklund HV, Boice JD Jr. Thyroid cancer after diagnostic doses of iodine-131: A retrospective cohort study. J Natl Cancer Inst 1988; 80: 1132-1138.

15. Manning MD, D'Angio GJ. Irradiation of the nasopharynx in children: A survey of patients 5-20 years after treatment for lymphoid hyperplasia. Clin Radiol 1967; 18: 173-175.

16. Kaplan MM, Boice JD Jr, Ames DB, Rosenstein M. Thyroid, parathyroid, and salivary gland evaluations in patients exposed to multiple fluoroscopic examination during tuberculosis therapy: A pilot study. J Clin Endocrinol Metab 1988; 66: 376382.

17. Fleiss JL. Statistical Methods for Rates and Proportions. New York: John Wiley; 1981.

18. Gart JJ. The comparison of proportions: A review of significance tests, confidence intervals and adjustments for stratification. Rev Int Stat Inst 1971; 39: 148-169.

19. Larsen PR. The thyroid. In: Wyngaarden JB, Smith LH, Jr, Eds. Cecil Textbook of Medicine. Phila: WB Saunders Co.; 1988: 1315-1338.

20. Rallison ML, Lotz TM, Bishop MA, Dobyns BM. Prevalence, incidence and regression of thyroid disease over a 20-year period in adolescents and young adults. American Thyroid Association (Abstract). Montreal; 1988.

21. McTiernan AM, Weiss NS, Daling JR. Incidence of thyroid cancer in women in relation to previous exposure to radiation therapy and history of thyroid disease. J Natl Cancer Inst 1984; 73: 575-581.

22. Ron E, Kleinerman RA, Boice JD Jr, LiVolsi VA, Flannery JT, Fraumeni JF Jr. A population-based case-control study of thyroid cancer. $\mathbf{J}$ Natl Cancer Inst 1987; 79: 1-12.

23. Vander JB, Gaston EA, Dawber TR. The significance of nontoxic thyroid nodules. Final report of a 15 -year study of the incidence of thyroid malignancy. Ann Intern Med 1968; 69: 537-540.

24. Phillips DF. Recalling former patients: Weighing the risks. J Am Hosp Assoc 1975; 49: 85-89.

25. Committee on the Biological Effects of Ionizing Radiations. The Effects on Populations of Exposure to Ionizing Radiation: 1980. Washington, D.C.: National Academy Press; 1980. 